\title{
SYMPOSIA TOPICS
}

\section{Prenatal Diagnosis and Treatment of CAH}

\section{7}

MOLECULAR PATHOLOGY OF 2l-HYDROXYLASE DEFICIENCY. T. Strachan, M. Tassabehji, S. Collier, P.J. Sinnott. Division of Human Genetics, University of Newcastle upon Tyne, Newcastle upon Tyne NEl 7RU, UK \& Dept. of Medical Genetics, University of Manchester, Manchester, UK.

Pathological mutations in steroid 21-hydroxylase deficiency appear to derive exclusively from sequence exchange between the 21-hydroxylase gene (CYP21B) and a closely related pseudogene (CYP2lA), located $30 \mathrm{~kb}$ telomeric to CYP2IB and functionally inactive because
several mutations distributed along its length. Three basic classes of disease chromosome are seen:

(a) deletion chromosomes where about $30 \mathrm{~kb}$ of DNA has been eliminated and a single 21-hydroxylase gene, often a CYP21A/CYP21B fusion gene, remains; (b) chromosomes which have 2 or more CYP21 genes, but without any apparent evidence for CYP21B-specific sequences (c) chromosomes which have 2 or more CYP21 genes, in which there is at least one gene with CYP21B-specific sequences, either a fusion gene or a mosaic, so-called "converted" gene. In the latter type of gene a central segment which may be very small or substantial shows CYP21A-specific sequences. Data will be presented on de novo mutations, showing how disease chromosomes can arise by unequal crossover and gene conversion-like events. The genotype-phenotype correlations will also be discussed.
9

HORMONAL MONITORING AND SIDE EFFECTS OF PRENATIAL DEXXMEIHASONE (DEX) TREATMFNT FOR 2l-HYMTOXYIASE DFFICIFNCY OONGENITAL ACRPNAL HYPERPLASTA (CAY). S Pang, A Clark, L Dolan, and D Schulman. Department of Pediatrics (SP, AC), University of Illinois College of Medicine, Chicago, IL 60612, USA, Children's Hospital Medical Center (LD), Cincinnati, OH 45229, USA, University of South Florida
college of Medicine (DS), Tampa, FL 33612, USA.

Maternal Dex therapy for fetal $\mathrm{CAH}$ at $1-1.5 \mathrm{mg}$ daily initiated between 5-8 weeks gestation and continued to birth, resulted in nomal genital outcome, mild virilization, and significant genital virilization in the three CAH newborns treated, and significant side effects in the three mothers, including excess weight gain (9-26 lbs greater at 35-37 weeks gestation compared to previous full-term pregnancies), glucose intolerance, facial hirsutism, and cushingoid features including moon-face, plethora, markedly discolored striae on arms, abdomen, breast, and thighs, which resulted in permanent on arms, abdomen, breast, and thighs, which resulted in permanent
scarring. In addition, hypertension, epigastric pain, and emotional
lability were noted, but not consistently. Amniotic fluid 17-OH Progesterone, androstenedione, and testosterone, were elevated in all three Dex treated cases. Matemal $E_{1}$ level was consistently suppressed in the case with the normal genital outcome, and were initially unsuppressed in the cases with mild and severe genital virilization. Conclusion: Dex therapy for prenatal treatment of CAH at a dose of $1-1.5 \mathrm{mg}$ daily was associated with significant maternal side effects. Amniotic fluid steroid levels may not be useful, while maternal serum $E_{1}$ level may be useful for therapeutic monitoring of fetal $\mathrm{CAH}$, this however requires further investigation.

\section{8}

PRENATAL DIAGNOSIS OF CONGENTTAL ADRENAL HYPERPLASIA DUE TO 21-HYDROXYLASE DEFICIENCY. Y. Morel, M. Murena, M.G. Forest, J.C. Carel, J. Leger, J-L Nivelon, M. David. Inserm U.329, Hôpital Debrousse, 69322 Lyon, Cedex 05 , France.

Prenatal diagnosis of the classic forms of the congenital adrenal hyperplasia (CAH) due to 21-hydroxylase deficiency has progressed at the same time as the advances made in the field of both endocrinology and molecular genetics. In 1975 , prenatal diagnosis of CAH was first made in the second trimester on the basis of raised levels of 17-hydroxyprogesterone (17-OHP) in the amniotic fluid (AF). Latcr an indirect prenatal diagnosis based on the genetic trackirig of CAH was greatly an indirect prenatal diagnosis based on the genetic tracking of CAH was greatly
facilitated by its linkage to HLA. By 1979 , to obviate the need for corrective genital facilitated by its linkage to HLA. By 1979 , to obviate the need for corrective genital
surgery the virilized genitalia in CAH female fetuses, a prenatal treatment was proposed by giving dexamethasone to the mother in early pregnancy, and later was shown to be successful. Rapidly, there was a demand for an early prenatal diagnosis of the affection. This was rendered possible by the advances in molecular biology associated with the practice of chorionic villus biopsy. Prenatal diagnosis was then done in the first trimester of the pregnancy, by either HLA typing using HLA-DNA probes or direct studies of the genetic abnormalities of the C4-CYP21 locus. In practice, four methods have been, and can still be used : measurement of AF levels of 17-OHP, HLA typing, molecular studies of the C4-CYP21 genes, all in association with fetal sex determination (measurement of $\mathrm{AF}$ testosterone levels, karyotype and more recently detection of $\mathrm{Y}$-chromosome specific sequences) karyotype and more recently detection of Y-chromosome specific sequences).
Steroid analysis remains a simple and safe method in the absence of prenatal Steroid analysis remains a simple and safe method in the absence of prenatal
treatment and the only informative method when there is no previous family study. Nowadays, an early and accurate prenatal diagnosis can be achieved by molecular genetic studies on DNA extracted from chorion villus samples, but it requires the previous study of the nuclear family. The choice between HLA typing and direct $\mathrm{C} 4$ CYP21 loci studies depend on the possibilities and expertise of a given laboratory. HLA typing is still more often used with the improvement of using molecular sndies instead of serological methods because almost all families are then informative. Nevertheless, HLA typing is unreliable in some circumstances: in case of a recombination event (1\% in our studies of over 250 families) or de novo mutation(s) and when the index case is deceased or not available. Studies of the C4-CYP21 gene locus by Southem blotting and the CYP21B gene mutations by PCR method have not these disadvantages. In our hands, Southern blotting study of the duplicated C4not these disadvantages. In our hands, Southern blotting study of the duplicated C4-
CYP21 region has shown some complex genetic rearrangements and is fully CYP21 region has shown some complex genetic rearrangements and is fully informative method for prenatal diagnosis in about $60 \%$ of CAH families. The 9
most common point mutations of the CYP21B gene causing CAH (and also present in the CYP21A pseudogene) can be detected after specific PCR amplification of the functional CYP21B gene. In these conditions, almost all families are informative in our experience. Moreover, using this direct genetic analysis associated with the possibility to detect the heterozygotes in a non related-CAH population, a prenatal diagnosis could be done in a family without a previous CAH affected child. A prenatal diagnosis in the first trimester is required in order to stop unnecessary prenatal treatments (if unaffected female fetuses and all males fetuses), or when the parents wish to interrupt the pregnancy in case of a $\mathrm{CAH}$ affected fetus. In our experience using molecular genetics of the CYP21-C4 loci ind sex determination by PCR method (SRY, Y-chromosome repeated sequences) or karyotype, prenatal $\mathrm{PCR}$ method (SRY, -chromolsome repeated sequences) or karyotype, prenatal
diagnosis has been successfully done in 23 pregnarcie, it tisk $1: 4$. In one case, diagnosis has been successfully done in 23 pregnarcies at risk 1:4. In one case, there was no previous family study because the index case was dead; in an other
family, the only informations were the state of heterozygoty of the parents: one being a relative of a characierized $\mathrm{CAH}$ family, the other being detected by a systematic ACTH test and confirmed by mutation studies.

\section{0}

PRENATAL TREATMENT OF CONGENITAL ADRENAL HYPERPLASIA (CAH) DUE TO 21-HYDROXYLASE DEFICIENCY : EUROPEAN EXPERIENCE IN 223 PREGNANCIES AT RISK. M.G. Forest and H.G. DOrr. on behalf of ESPE. PREGNANCIES AT RISK. M.G. Forest and H.G. Dorr. on behalf of ESPE.
INSERM-U.329, Hôpital Debrousse, 69322 Lyon Cedex 05, France and Klinik für Kinder und Jugendliche der Universitắ Erlangen-Nümberg, 8520 Erlangen, Germany. In the aim to prevent the in utero virilization of $\mathrm{CAH}$ affected females a prenatal treatment $(\mathrm{Rx})$ has first been attempted by David and Forest in 1979; later these authors reported their first results and proposed a therapeutic protocol (J. Pediatr. 1984; 104: 799-803). Except for the experience of the French multicentric study in 42 pregnancies at risk (Endocr. Res.1989;15:277-301), prenatal Rx of CAH using therapeutic protocols quite similar to the above, and subsequently reported in the literature were concerned with a limited number of cases. Indeed, prenatal $\mathrm{Rx}$ of $\mathrm{CAH}$ is not frequently instituted. Nevertheless, a number of experiences have not been reported. This is why, we decided to collect a maximum of data from European Pediatricians. A questionnaire was to collect a maximum of data from European Pediatricians. A questionnaire was
constructed to enquire about the effects of prenatal Rx on the fetus on one hand constructed to enquire about the effects of prenatal $\mathrm{Rx}$ on the fetus on one hand
(therapeutic protocols, prenatal diagnosis $(\mathrm{Dg})$, results on the extemal genitalia of $\mathrm{CAH}$ female fetuses, effects if any on the development of the fetus or child) and on the mother on the other hand (follow-up, compliance and tolerance of $\mathrm{Rx}$, course of pregnancy, short and long term effects or side-effects in the mothers). Informations were obtained in a total of 223 treated pregnancies: 75 cases from the French multicentric study (M.G. Forest, M.David), 58 German cases (collected by H.G. Dorr and W.G. Sippell in cooperation with the APE), and 27 Scandinavian cases coordinated by E.M. Ritzen. The questionnaire was also sent to the other European ESPE Members and to date data were collected on 63 additional cases. There was no prenatal Dg in 20 cases (not available or refused). Prenatal Dg was first made on HLA typing and/or steroid measurement in the amniotic fluid. An earlier prenatal Dg was subsequently made by HLA typing on chorion villus sampling (CVS). On the whole, steroid measurement was safe (if Rx interrupted) except in 2 cases and HLA serological typing helpful, but at least 8 false prenatal Dg
were recorded. For the last 2-4 years, DNA studies are performed on CVS (HLA RFLP, C4-CYP21 genes) with increasing safety. Prenatal $R x$ was with dexamethasone (Dex) or Dex acetate (France in particular), usually at a dose of $0.5 \mathrm{mg}, 2$ to 3 times a day. The Dex dose was more rarely adapted to maternal body weight $(20 \mu \mathrm{g} / \mathrm{kg}$, as advocated by Forest et al (above ref.). Among the $38 \mathrm{CAH}$ treated females, 37 were born, with normal (9) or slightly virilized genitalia (17); none of them would need surgery. Prenatal $R x$ was not successful (stage Iu//V) for apparently logical reasons (late start of $R x$, insufficient Dex dose, early interruption, lack of compliance) in 9 CAH females but not explained in 2 others. There was a remarkable lack of side-effects in feruses or children, except for a 2 others. There was a remarkable lack of side-effects in fetuses or children, except for a
severe hydrocephalus in a male fetus (treated for $7 \mathrm{wks}$ ) and 2 deaths in utero near term while Rx was stopped for several months (both not attributed to Dex Rx by specialists), and an unexplained failure to thrive in a CAH girl treated until term. Matemal acceptance of $\mathrm{Rx}$ was said to be good to excellent. Maternal compliance was usually good (as judged by low maternal levels of cortisol, DHAS or estriol). Matemal side-effects were reported in some but not all mothers. In the mothers where $\mathrm{Rx}$ was stopped in midpregnancy (unaffected cases) there was often no complaint or that of an excessive weight gain (reasonably controlled by salt and caloric restriction in the French study), observed more often in the mothers given $1.5 \mathrm{mg}$ of Dex, and always resuming after delivery. When treatment was continued until term $(n=53)$, more serious side-effects were observed in some mothers : marked weight gain $(n=15)$, edema $(n=3)$, increased blood pressure $(n=5)$. In addition, $R x$ tolerance was poor in 7 mothers (weight gain, edema, fatigue, feeling of exhaution, or hypertension, or gestational diabetes mellitus) leading twice to internupting Rx despite prenatal Dg of a CAH female fetus. This is why a more detailed follow-up of the maternal well-being is undertaken. In conclusion, whenatal $\mathrm{Rx}$ detaled follow-up of the maternal well-being is undertaken. In conclusion, prenatal Rx
of $\mathrm{CAH}$ appears effective, providing a good rationale in the Rx protocol. Observations of CAH appears effective, providing a good rationale in the Rx protocol. Observations
made in a large number of pregnancies show that short term Rx (started at $4-7$ wks, and stopped after a prenatal Dg based on DNA studies on CVS, at about $12 \mathrm{wks}$ ) is usually well tolerated. Tolerance of long term $\mathrm{Rx}$ seems to be a problem in some mothers. These findings lead us to propose that Dex Rx should be adapted to matemal size in all mothers $(20 \mu \mathrm{g} / \mathrm{kg})$, then Dex dose should or could eventually be decreased by $50 \%$ from mid$\mathrm{S} 3$ pregnancy to term in those mothers who bear a $\mathrm{CAH}$ female fetus. 